\title{
Chaotic Dynamics of a Linear Chain of Periodically Stimulated Neurons with Random Synaptic Connections
}

\author{
R.A. Kosiński ${ }^{a, b}$, A. KRAWIEcki ${ }^{a}$ AND A. Sukiennicki ${ }^{a, c}$ \\ ${ }^{a}$ Faculty of Physics, Warsaw University of Technology \\ Koszykowa 76, 00-662 Warsaw, Poland \\ ${ }^{b}$ Central Institute for Labor Protection \\ Czerniakowska 16, 00-701 Warsaw, Poland \\ ${ }^{c}$ Deptartment of Solid State Physics, University of Łódź \\ Pomorska 149/153, 90-283 Łódź, Poland
}

(Received March 19, 2001)

\begin{abstract}
Dynamics of a neural network in the form of a linear chain of artificial neurons $S_{i} \in(-1,1)$ influenced by an external sinusoidal stimulation is investigated as a function of the range $k$ of synaptic connections with random values. Time evolution of the network is periodic for small $k$, however, clusters of neurons oscillating with a triple period of external stimulation, with quasiperiodic or with chaotic time evolution may occur. For increasing $k$ the number and width of the chaotic clusters increase and for $k>4$ the chaotic motion occurs in the whole network. A route to chaos in the considered system is discussed.
\end{abstract}

PACS numbers: 87.10.+e, 05.45.-a

\section{Introduction}

Artificial neural networks (ANNs) are a kind of complex systems which exhibit such phenomena like chaos (see e.g. [1, 2]), quasiperiodic evolution [3, 4], intermittency $[5,6]$, synchronization $[3,7]$ and stochastic resonance [8-10]. A particular role plays chaotic time evolution, which may occur in different kinds of ANNs, because it resembles the time evolution of the brain [11]. For this brain activity the name stochastic chaos has been recently adopted [12]. Besides, a number of interesting applications may be based on chaotic evolution of neural networks [13], e.g., information processing [14], optimization [15] and memory properties [16]. There- 
fore, the number of papers investigating physical properties of ANNs connected with chaos, like controlling chaos [17], pattern formation [18], necessary conditions for the occurrence of chaotic time evolution of the network [8, 19], routes to chaos $[8,19]$, etc., has recently increased. In the majority of papers, however, no periodic stimulation was used.

In our work the dynamics of a linear chain of periodically stimulated artificial neurons with random synaptic connections, limited to $2 k$ nearest neighbors, is investigated numerically. A route to chaos and both local (referring to a single neuron embedded in a chain) and global (referring to the whole network) dynamical properties of the system are discussed. An application of periodic stimulation to this system results in a route to chaos via formation and growth of clusters in which oscillations with a triple period, quasiperiodic and, finally, chaotic oscillations appear.

\section{The model}

Our ANN has a form of a linear chain consisting of $N$ neurons $S_{i}$, for which current states are as follows:

$$
S_{i}(t+1)=\tanh \left[g h_{i}(t)+I(t)\right],
$$

where

$$
h_{i}(t)=\sum_{j=i-k}^{i+k} J_{i j} S_{j}(t),
$$

and $h_{i}$ is the local field acting on $i$-th neuron in the discrete time $t, J_{i j}$ is the synaptic connection between $i$-th and $j$-th neuron, $g$ is the gain parameter and $I(t)=A \sin (2 \pi t / T)$ is the external stimulation acting on each neuron. The synaptic connections of $i$-th neuron with its $2 k$ nearest neighbors and itself $\left(J_{i i}\right)$ have random values $J_{i j} \in[-1,1]$. Periodic boundary conditions are used. Synchronous updating of the states of neurons is used for the calculation of the time evolution of the network. As a starting configuration $\left\{S_{i}(0)\right\}$ random values of all neurons $S_{i} \in(-1,1)$ were mostly assumed. Sometimes, the uniform initial conditions $\left\{S_{i}(0)=0\right\}$ were also used.

An analytic investigation of the neuron system presented here is not possible, because the system is too large. Therefore, numerical simulations of the dynamics of the system were performed. For the investigation of the local network dynamics, i.e., dynamics of a single neuron embedded in the chain, profiles of the states of all neurons $S(i)$ for different times and return maps $S_{i}(t+T)[S i(t)]$, showing the character of the Poincaré sections, were calculated, while for the observation of the global dynamical properties of the whole network average deviation

$$
\sigma(t)=\frac{1}{N} \sum_{i=1}^{N}\left[S_{i}(0)-S_{i}(t)\right]^{2}
$$

and the return map $\sigma(t+T)[\sigma(t)]$ were used. 
Numerical experiments were made for $N=151$, gain parameter $g=1$, amplitude and period of external stimulation $A=1$ and $T=24$, respectively. The behavior of the network for other values of these parameters was also monitored. For each value of the control parameter $k$, calculations were repeated for 10 different distributions of $[J]$ and 10 initial states of the network $\left\{S_{i}(0)\right\}$, coming from a random number generator.

The kind of the time evolution of the network was observed as a function of the number of neighbors, starting with $k=1$, which corresponds to the most diluted synaptic connections, up to a fully connected network with $k=N$, which corresponds to the network of Hopfield type, i.e., the network with no internal geometric structure.

\section{Results and discussion}

Generally it was found that for a strong dilution of synaptic connections purely spatial chaos occurs in the chain. The time evolution of the network follows the external stimulation, i.e., it is periodic with the period $T$, although the network is spatially disordered. For an increasing number and range of synaptic connections the network dynamics becomes more complex and for $k$ large enough time evolution of the whole network is chaotic, i.e., spatiotemporal chaos appears. Typically, spatiotemporal chaos occurs via the formation of clusters of neurons which show periodic (with a triple period), quasiperiodic, and chaotic oscillations. The number and size of the clusters increase with $k$.

Let us illustrate the above-mentioned rules with several examples. For $k=1$, the global dynamics of the network, described by $\sigma(t)$, is shown in Fig. 1a.
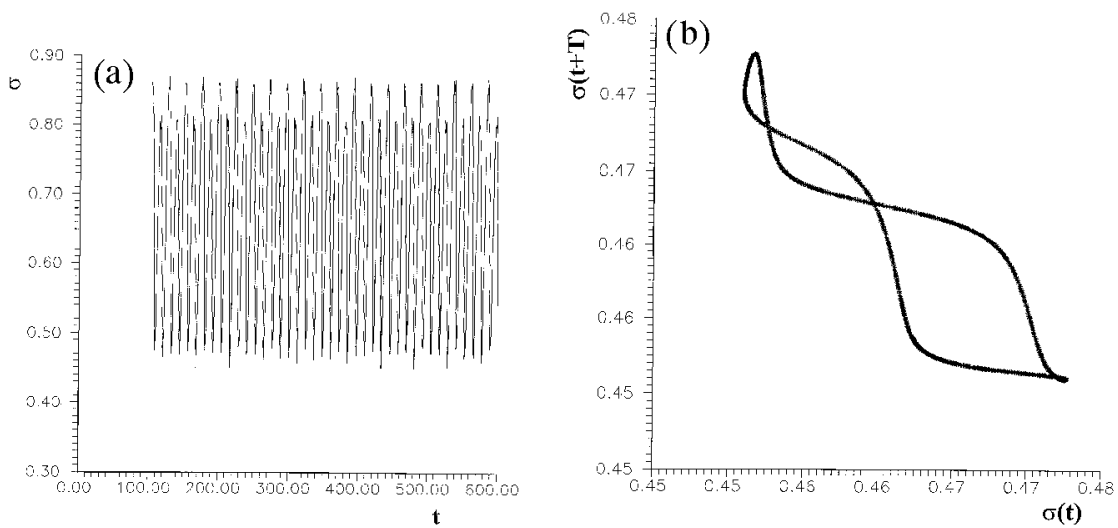

Fig. 1. (a) Average deviation $\sigma(t)$ as a function of time and (b) return map $\sigma(t+T)[\sigma(t)]$ for the chain with $k=1$. 

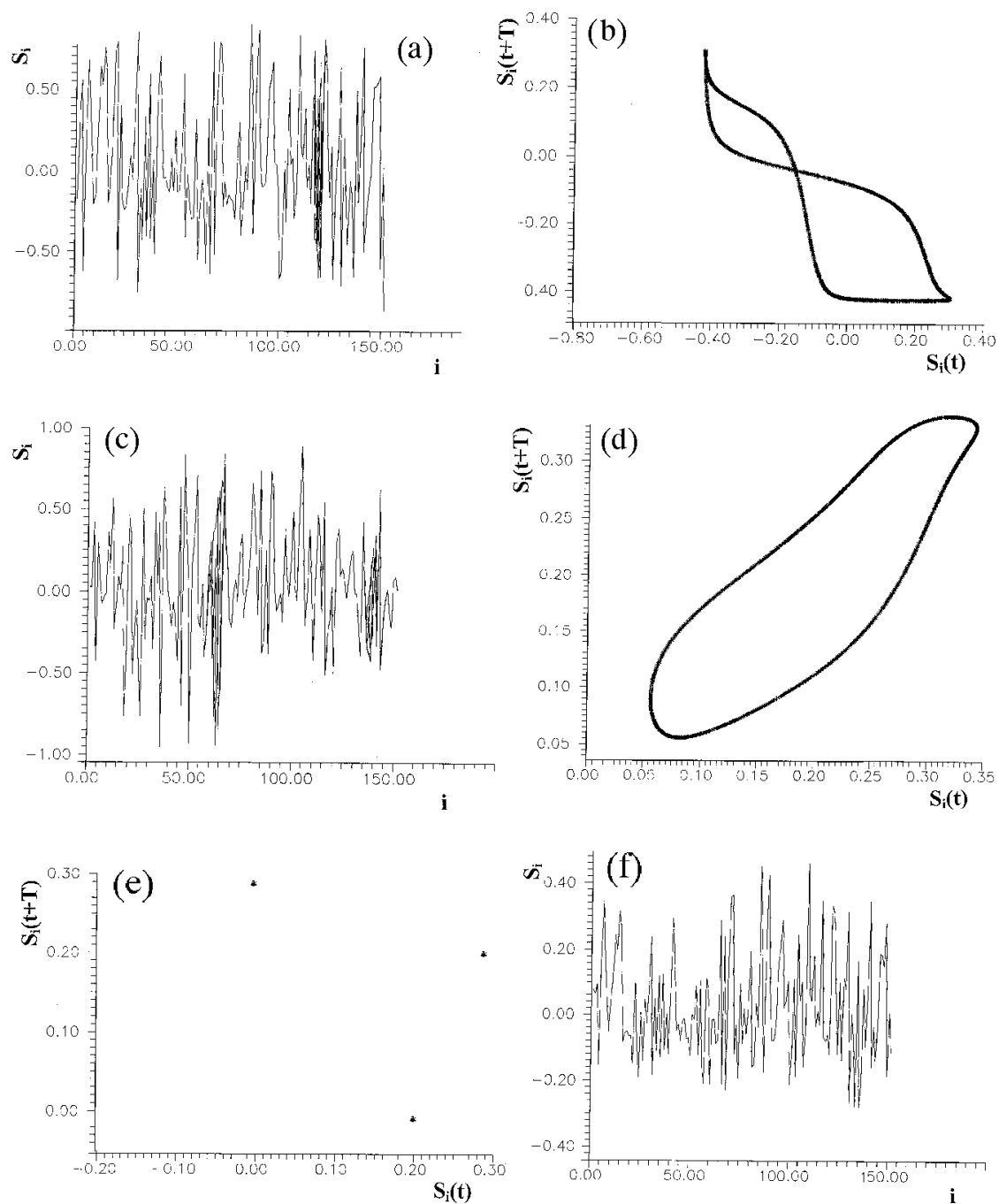

Fig. 2. (a) Profiles of the neurons $S(i)$ in the chain for $t=\Delta t, 2 \Delta t, 3 \Delta t, \ldots, 10 \Delta t$ (where $\Delta t=40 T=960$ time steps) superimposed, show the cluster of approximately 12 neurons, lying in the neighborhood of 122-nd neuron (calculations with $\Delta t=T$ give the same result). (b) Return maps $S_{i}(t+T)\left[S_{i}(t)\right]$ for the neuron $i=117$ from the cluster shown in (a) indicates quasiperiodic evolution. (c) Profiles $S(i)$ in the chain depicted as in (a), but with uniform initial states of the neuron. Two clusters lying near the neurons $i=60$ and $i=140$ are seen. (d) Return map $S_{i}(t+T)\left[S_{i}(t)\right]$ for the neuron $i=140$ from the right cluster shown in (c) and (e) for the neuron $i=60$ from the left cluster. (f) Profiles $S(i)$ in the chain depicted as in (a), but with the gain parameter $g$ reduced to $g=0.5$. 
It has an almost periodic character. However, a more exact inspection of this time evolution, identified by the return map $\sigma(t+T)[\sigma(t)]$, shows that in a small scale the time evolution is quasiperiodic (Fig. 1b). This return map proves that the phase trajectory lies on a surface of a two-dimensional torus corresponding to two incommensurate frequencies present in the system. An observation of the local dynamical properties of the network (Fig. 2a) indicates that a cluster of approximately 12 neurons, lying in the neighborhood of 122-nd neuron, does not follow the external stimulation. The cluster can be revealed in the superimposition of a number of profiles $S(i)$ calculated for the times $t=n \Delta t$, where $n=1,2,3, \ldots$ and $\Delta t$ is the multiple of the period $T$ of external stimulation. In the case of periodic evolution of the chain with the period $T$ the curves $S(i)$, calculated for $\Delta t=T$, overlap in the whole chain. On the other hand, when the time evolution of the chain is irregular, curves $S(i)$ for arbitrary $\Delta t$ do not overlap in these segments (clusters of neurons) of the chain which do not follow the external stimulation. The return map $S_{i}(t+T)\left[S_{i}(t)\right]$ for the neuron $i=117$, one of the neurons lying inside the cluster, shows that the neurons in the cluster move quasiperiodically (Fig. 2b). Depending on initial conditions and current distribution of synaptic connections 0,1 or 2 clusters with dynamics other than that of external stimulation can be observed in the network. Varying other parameters exerts also a significant influence on the network dynamics. For example, for the uniform initial conditions, $\left\{S_{i}=0\right\}$ at $t=0$, two clusters lying near the neurons $i=60$ and $i=140$ (Fig. 2c) are observed in the network. Investigations of the local dynamics of the network in this case shows that in the first cluster quasiperiodic evolution occurs (Fig. 2d), while in the second one periodic evolution with the periodicity $3 T$ is observed (Fig. 2e). On the other hand, decreasing the gain parameter from $g=1$ to $g=0.5$, with the same values of other parameters, leads to a simplification of the dynamics of the network - only purely periodic dynamics of the whole network is observed (Fig. 2f), i.e., all neurons oscillate following the oscillations of the external stimulation $I(t)$.

For $k=2$ a number of clusters may occur in the network too and the case with pure periodic evolution was not found. Generally speaking, a width of the clusters increases. Figure 3a shows an example of a cluster with the approximate width of 27 neurons, lying in the neighborhood of 113-th neuron. The return map of one of neurons with dynamics typical of this cluster - with $i=115$ - indicates that the time evolution of neurons in this cluster is periodic, with the period $3 T$ (Fig. 3b).

For $k=3$ the global dynamics of the network observed at $\sigma(t)$ looks like chaotic (Fig. 4a), however, the shape of the return map $\sigma(t)[\sigma(t+T)]$, shown in Fig. 4b, indicates a certain form of intermediate type of time evolution between quasiperiodic and chaotic. This return map reveals the process of decay of the two-dimensional torus which is characteristic of the quasiperiodic route to chaos. The observation of the local dynamical properties shows, e.g., two clusters 

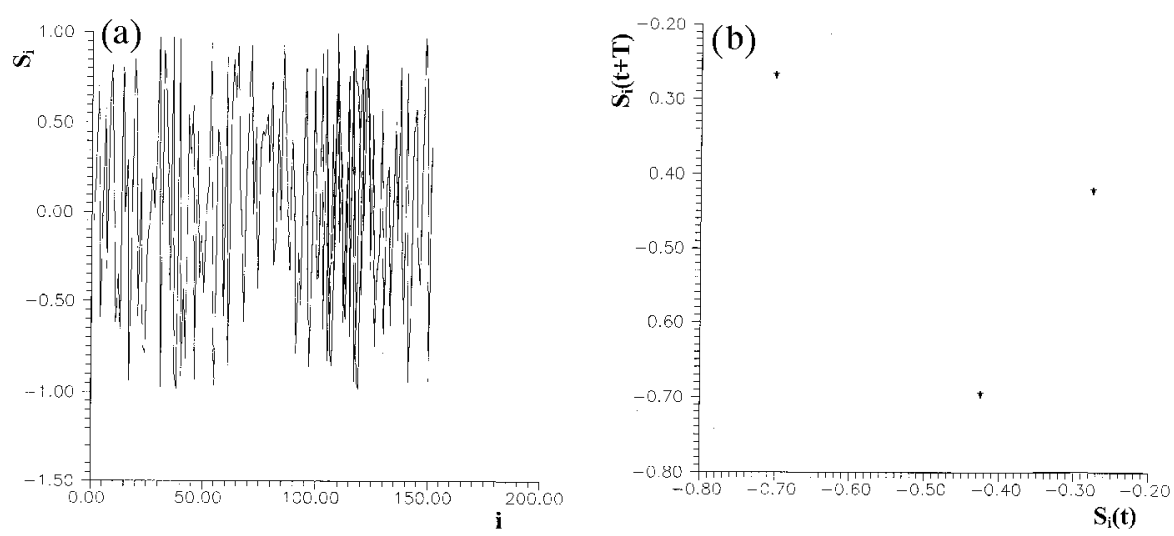

Fig. 3. (a) Profiles $S(i)$ for $k=2$; cluster of neurons near the neuron $i=113$ is seen; curves for the same times $t$ as in Fig. 2a. were superimposesd. (b) Return map $S_{i}(t+T)\left[S_{i}(t)\right]$ for the $i=113$ neuron.
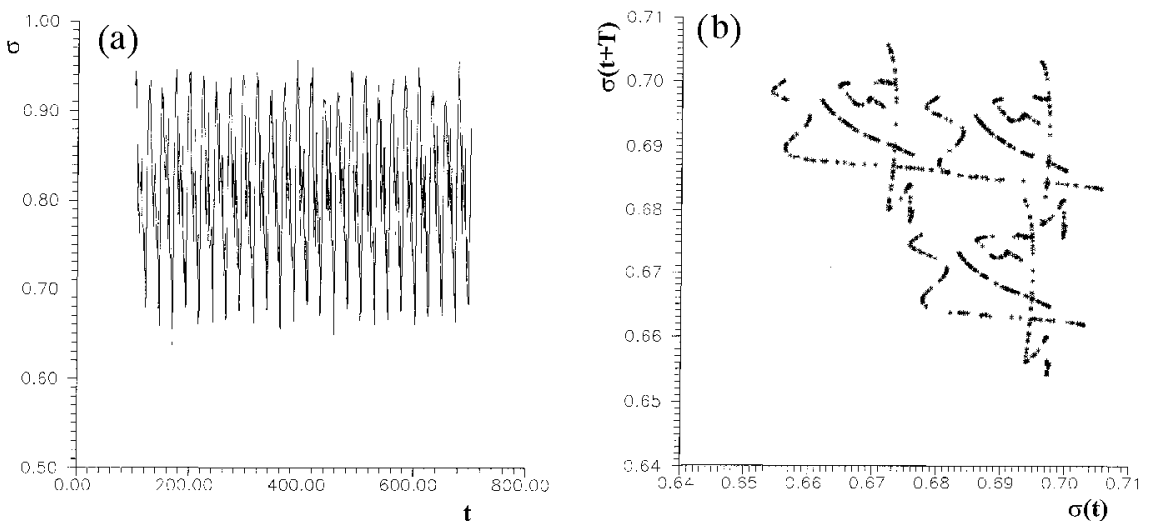

Fig. 4. (a) Average deviation $\sigma(t)$ and (b) return map $\sigma(t+T)[\sigma(t)]$ for the chain with $k=3$.

(Fig. 5a) lying near neurons $i=100$ and $i=15$. In the first of these clusters evolution with the period $3 T$ is observed (Fig. 5b). In the second one, however, the evolution should be again interpreted as "not fully developed chaos" rather than the quasiperiodic behavior (Fig. $5 \mathrm{c}$ ).

For $k=4$ chaotic time evolution is observed in the whole network (see Fig. 6a,b). A fully disordered form of the return map (Fig. 6b) is typical of chaotic motion. Comparison between the return maps $\sigma(t+T)[\sigma(t)]$ for $k=3$ and $k=4$ (Figs. $4 \mathrm{~b}$ and $6 \mathrm{~b}$ ) justifies the interpretation of the network time evolution in the case of $k=3$ as intermediate between quasiperiodic and chaotic. Local dynamics of the network is also chaotic, however, return maps of different neurons may be different (see Fig. 7a,b,c,d), and some of them still have an intermediate character, like in Fig. $7 \mathrm{~b}$. 

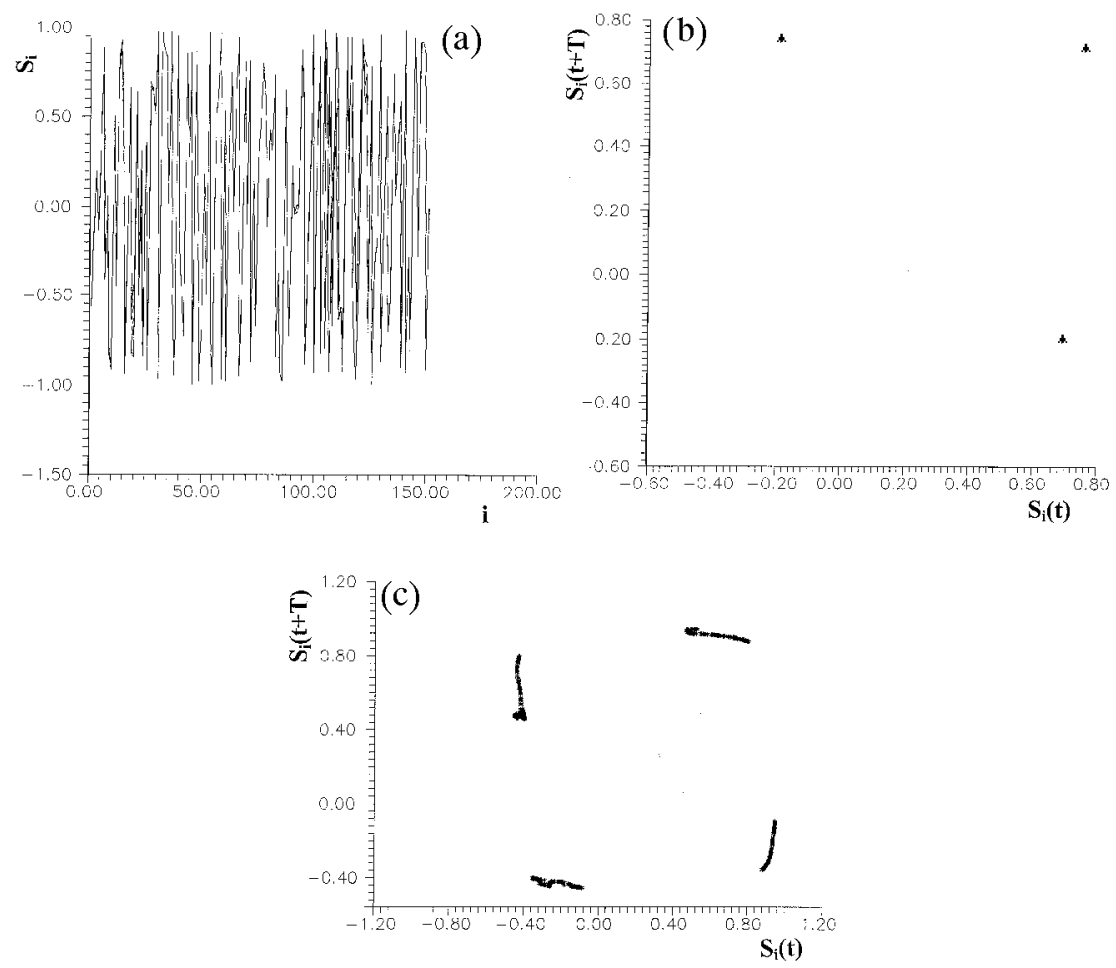

Fig. 5. (a) Profiles $S(i)$ for $k=3$; curves for the same times $t$ as in Fig. 2a were superimposed. (b) Return map $S_{i}(t+T)\left[S_{i}(t)\right]$ for the neuron $i=15$ from the left cluster and (c) for the neuron $i=100$ from the right cluster.
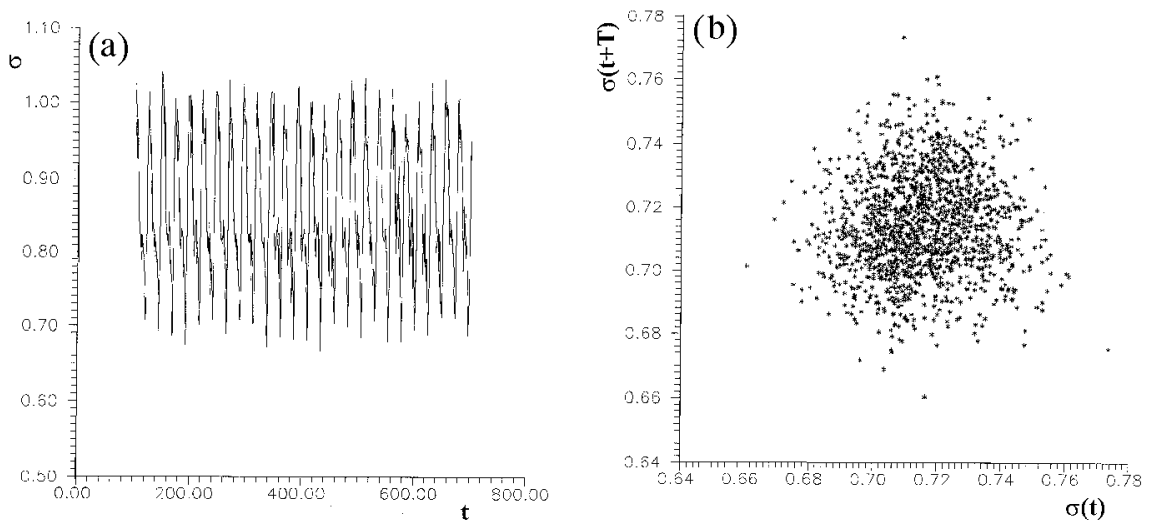

Fig. 6. Chaotic motion of the network for $k=4$. (a) Average deviation as a function of time $\sigma(t)$. (b) Return map $\sigma(t+T)[\sigma(t)]$. 

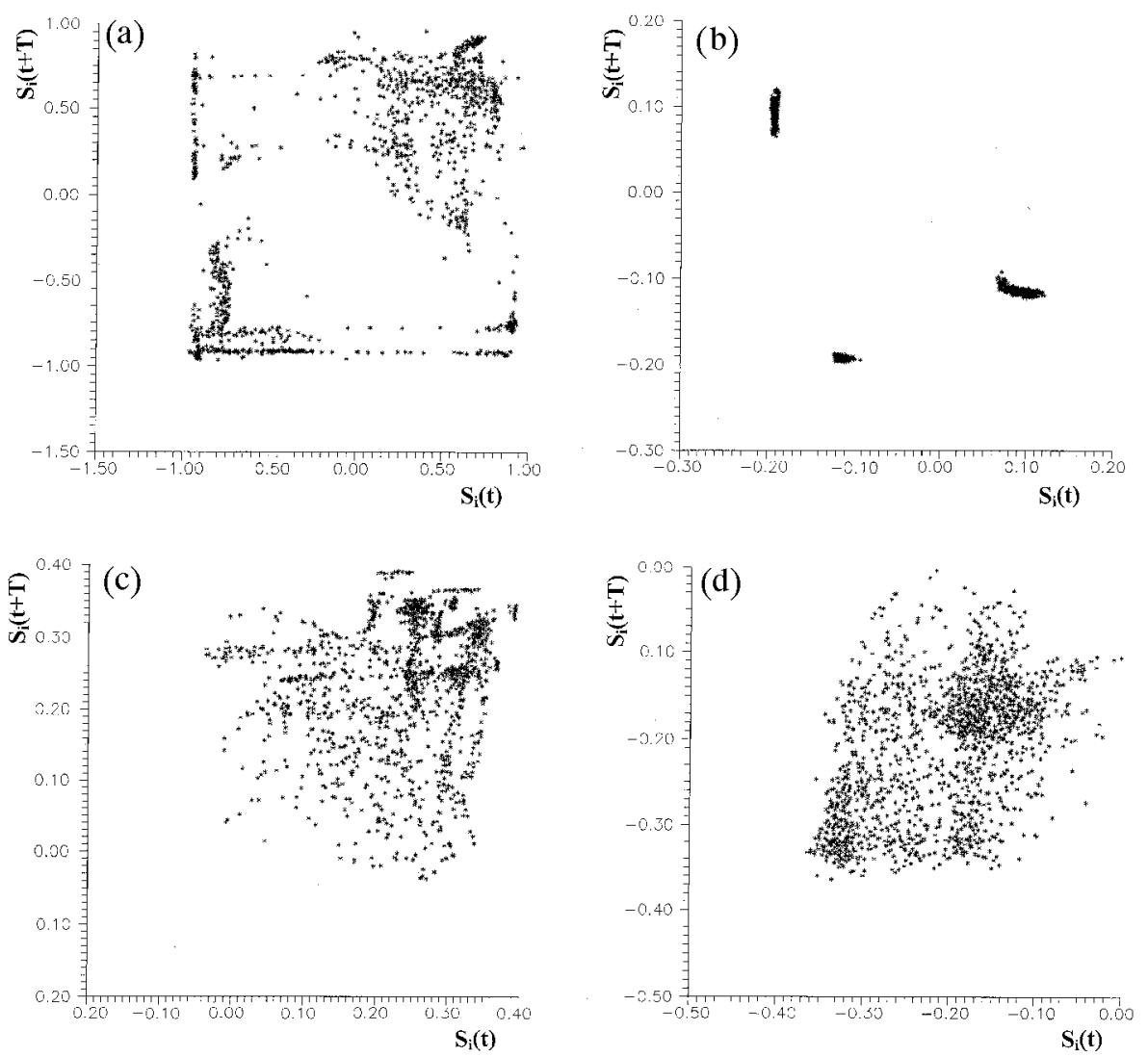

Fig. 7. Local dynamical properties of the network for neurons $i=40,75,106,150$ are shown in (a), (b), (c), and (d), respectively. An intermediate type of dynamics for a neuron with $i=75$ can be seen.

For $k=5$ the chaotic cluster incorporates all neurons, as is shown at profiles $S(i)$ (Fig. 8a). In this case the return maps of all neurons have a fully disordered character (Fig. 8b) - the intermediate type of neuron's evolution disappears. Thus, it can be concluded that for $k \geq 5$ the fully developed chaotic evolution both in space and time occurs in the whole network.

As we see in our system the route from the periodic to chaotic evolution occurs via the destabilization of oscillations of certain neurons in the chain, in the neighborhood of which clusters of neurons with similar time evolution, but different than that of external stimulation, appear. This evolution may have a quasiperiodic as well as a periodic character, with the period 3T. A position of these destabilized neurons depends on the current realization of the random initial state of the network and the random distribution of synaptic connections. When the density of synaptic connections increases, widths and number of the clusters 

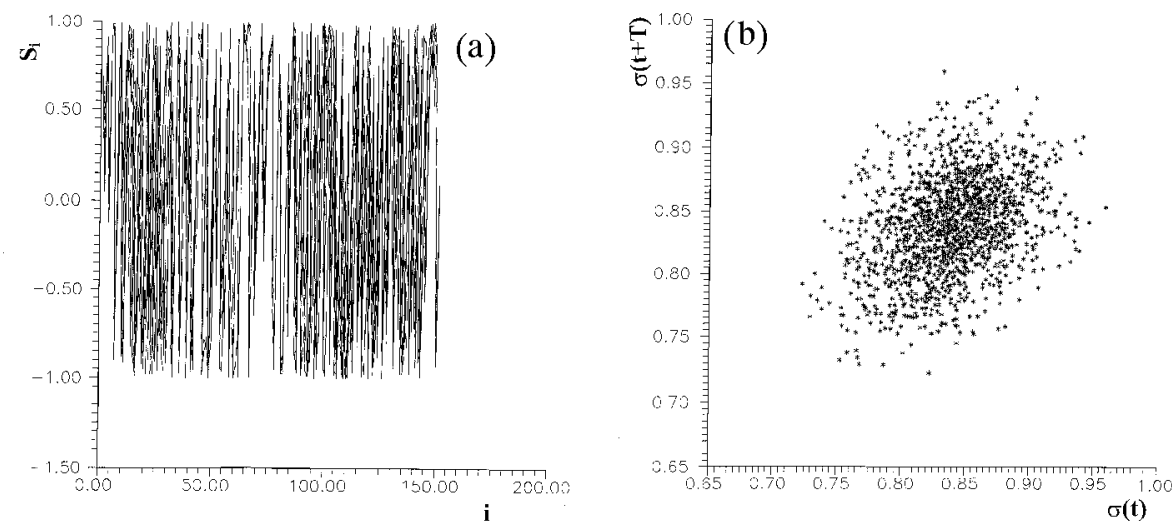

Fig. 8. For $k=5$ cluster of neurons with chaotic motion incorporates the whole chain. (a) Profiles $S(i)$ for the same times $t$ as in Fig. 2a were superimposed, (b) return map $\sigma(t+T)[\sigma(t)]$.

increase also. In some of these clusters chaotic evolution occurs. The number of neurons with such evolution increases and for $k \geq 4$ chaotic evolution covers the whole network. It means that the network with random synaptic connections dense enough, in particular with full connectivity $(k=N)$, as in the Hopfield network, is prone to chaotic evolution. Such an increase in susceptibility of the network to chaotic evolution with the increase in the density of synaptic connections was reported earlier for different types of neural networks [7,4].

The route to chaos via quasiperiodicity is one of the main routes to chaos as observed earlier in a number of dynamical systems, in particular in a simple neural system with $N=2$ neurons by Wang [20] and in a greater network with random synaptic connections by Albers, Sprott and Dechert [19]. Another route to chaos by period doubling - was also frequently observed in neural networks of different kinds [21-23]. In our investigations, however, instead of the period doubling the route to chaos by the increase in the period of motion of certain neurons to the value $3 T$ was found. This behavior corresponds to Li-Yorke theorem known as "period 3 implies chaos" [24].

Thus, it can be stated that the route to chaos of a linear chain of neurons has a mixed character - by quasiperiodic evolution or by an increase in the period of oscillations to $3 T$. The current realization of the route to chaos is determined by the choice of random initial state of the network and the choice of the distribution of random synaptic connections.

The value of $k$, defining the lowest limit of the chaotic evolution of the network, is influenced by the values of the gain parameter $g$ (cf. Fig. 2a and Fig. 2f), the amplitude $A$ of the external stimulation and the length of the chain $N$. Generally speaking, decreasing the value of $g$ and increasing the amplitude $A$ shift the lowest limit of chaotic motion to greater values of $k$. Such an influence of 
the gain parameter $g$ on the limit of chaotic motion was observed also in [4, 22]. Decreasing the length $N$ of the chain also increases the lowest limit of chaotic behavior.

\section{Conclusions}

It was found that the linear chain of neurons with random synaptic connections and external sinusoidal stimulation has complex dynamical properties, including different forms of periodic oscillations, quasiperiodic and chaotic time evolution. The increase in the number of synaptic connections per neuron, which results in the disappearance of the internal geometrical structure of the network, leads from periodic to chaotic time evolution. The route to chaos observed in the chain may be different for its different segments, depending on the initial states of the neurons and current distribution of synaptic connections. In particular, the route to chaos by the occurrence of oscillations with a triple period was found.

\section{References}

[1] H. Sompolinsky, A. Crisanti, Phys. Rev. Lett. 61, 259 (1988).

[2] M. Adachi, K. Aihara, Neural Netw. 10, 83 (1997).

[3] A. Tonnelier, S. Meignen, H. Bosch, J. Demongeot, Neural Netw. 12, 1213 (1999).

[4] R.A. Kosiński, A. Jaroszewicz, Chaos, Solitons and Fractals 9, 1481 (1998).

[5] J.E. Moreira, D.M. Auto, Europhys. Lett. 21, 639 (1993).

[6] A. Krawiecki, R.A. Kosiński, Int. J. Bifurcation and Chaos Appl. Sci. Eng. 9, 97 (1999).

[7] D. Hansel, H. Sompolinsky, Phys. Rev. Lett. 68, 718 (1992).

[8] R. Riani, E. Simonotto, Phys. Rev. Lett. 72, 3120 (1994).

[9] A. Krawiecki, A. Sukiennicki, R.A. Kosiński, Int. J. Mod. Phys. B 14, 837 (2000).

[10] A. Krawiecki, A. Sukiennicki, R.A. Kosiński, Phys. Rev. E 62, 7683 (2000).

[11] A. Babloyantz, J.M. Salazar, Phys. Lett. A 111, 152 (1985).

[12] W.J. Freeman, Neural Netw. 13, 11 (2000).

[13] Neural Networks and their Applications, Ed. J.G. Taylor, Wiley, Chichester 1996.

[14] S. Ishii, K. Fukumizu, S. Watanabe, Neural Netw. 9, 25 (1996).

[15] I. Tokuda, T. Nagashima, K. Aihara, Neural Netw. 10, 1673 (1997).

[16] T. Nagashima, in: Proc. Int. Symp. on Nonlinear Systems, NOLTA93, Hawaii, 1993, IEEE Soc. Press, Hawaii Section, Honolulu 1993, p. 2.7-4.

[17] T. Saito, K. Mitsubori, in: Proc. Int. Symp. on Nonlinear Systems, NOLTA93, Hawaii, 1993, IEEE Soc. Press, Hawaii Section, Honolulu 1993, p. 1.3-4.

[18] M. Usher, M. Stemmler, Z. Olami, Phys. Rev. Lett. 74, 326 (1995).

[19] D.J. Albers, J.C. Sprott, W.D. Dechert, Int. J. Bifurcations and Chaos Appl. Sci. Eng. 8, 1463 (1998). 
[20] X. Wang, in: Proc. Int. Joint Conf. on Neural Networks, Seattle 1991, IEEE New York Press, New York 1992, p. 91.16-91.2.

[21] N. Lemke, J.J. Arenzon, R.M.C. Almeida, S. Goulart Rosa Jr., J. Phys. A, Math. Gen. 28, 1335 (1995).

[22] E.K. Blum, X. Wang, Neural Netw. 5, 577 (1992).

[23] S.K. Han, S.H. Park, T.G. Yim, S. Kim, S. Kim, Int. J. Bifurcations and Chaos Appl. Sci. Eng. 4, 877 (1997).

[24] T.Y. Li, J.A. Yorke, Am. Math. Monthly 82, 985 (1975). 